\title{
Airway Metrics, Anatomy and Growth Performance of Pigs Reared Indoors and Outdoors
}

\author{
${ }^{1,2}$ Chakia Joi McClendon, ${ }^{1}$ Sherrell Pettiford, ${ }^{1}$ Dawn Conklin, \\ ${ }^{1}$ Lauren Kloc, ${ }^{1}$ Sang-Hyon Oh and ${ }^{1}$ Jenora Turner Waterman \\ ${ }^{1}$ Department of Animal Sciences, North Carolina Agricultural and Technical State University, \\ 1601 East Market Street, Greensboro, North Carolina 27411, USA \\ ${ }^{2}$ Department of Energy and Environmental Systems, North Carolina Agricultural and Technical State University, \\ 1601 East Market Street, Greensboro, NC, USA
}

Received 2013-09-18, Revised 2013-09-21; Accepted 2013-09-24

\begin{abstract}
While the swine industry has made incredible strides to improve ventilation, air quality in confinement buildings remains an issue. The goal of this study is to characterize correlation between large airway metrics and growth performance in piglets reared indoors and outdoors on pasture. Three experimental trials were conducted: Trial 1 included three breed types that were strictly raised indoors, Tamworth X Berkshire, Berkshire X Berkshire and Hereford X Berkshire $(\mathrm{n}=4-5$ each). Trials 2 and 3 consisted of animals reared in both environments; trial 2 had 28 pigs $(n=14$, indoor and $n=14$, outdoor) and trial 3 had 48 pigs $(n=24$, indoor and $n=24$, outdoor). For trial 3 , body weights were recorded weekly for seven weeks to adjust tracheal measure for body size. Total tracheal and lumen diameters were determined for all animals on trial. Histological evaluations were performed to evaluate potential differences among indoor and outdoor pig populations. One-way and two-way Analyses of Variance (ANOVA) were performed and LS means with the PDIFF option was used to separate means as applicable. Animals reared indoor shad significantly larger tracheal diameters and lumens compared to those reared outdoors ( $p$-value $<0.05$ ). Outdoor animals had a larger variation of body weights than indoor animals; however, no correlation between tracheal measurements and body weights was present. Histological evaluation of airway sections revealed a 1.4 fold increase in the total population of goblet cells in tracheas of indoor pigs (pvalue $<0.0152$ ) versus outdoor pig tracheas. Taken together, subtle differences may exist in airways of pigs reared indoors versus outdoors; however, airway distinctions do not appear to effect growth performance of piglets.
\end{abstract}

Keywords: Airway Measurements, Tracheal Diameter, Pig, Production, Growth Performance

\section{INTRODUCTION}

Swine production is a multi-billion dollar global industry and pork accounts for $36 \%$ of world meat intake, making it the most consumed meat, followed by poultry (33\%) and beef (24\%) (FAO, 2010). Therefore, to accommodate for the increase in demand for pork, swine production has changed from traditionally raising swine outdoors to a more intensive production system. In the past, swine were traditionally raised outdoors where they were permitted to roam the land and forage as they please. Even though niche markets still raise animals outdoors in a free-range environment, to increase production, swine are now primarily produced in largescale Concentrated Animal Feeding Operations (CAFO). CAFO-style facilities are attractive in that they allow producers to utilize a series of production steps, automatic feeders and waste management 1601 East Market Street, Greensboro, North Carolina 27411, USA Tel: 336-285-4815 Fax: 336-334-7288 
processes within environmentally controlled buildings; however, air quality issues remain a challenge. Research has shown a high degree of accumulation of gases, dust and endotoxins within swine confinement housing (Cole et al., 2000; Von Essen and Auvermann, 2005) which have been linked to the development of respiratory symptoms in workers and farm visitors (Poole et al., 2007; Radon et al., 2007). Although substantial research has been conducted regarding these two issues, less attention has been directed towards understanding large airway dynamics of pigs reared within both environments.

Due to similarities in respiratory anatomy and physiology among pigs and people, human respiratory health research studies may provide insight for understanding physiological processes within porcine airways and the reverse is also likely to be true. The trachea, also called the windpipe, is a large tube which connects to the pharynx and larynx (voice box) and subdivides into two bronchi as it enters the lungs, thus allowing the passage of air. The trachea is lined with a mucus-secreting mucosal barrier which serves as a first line of defense against particulates which enter the airway. Consequently, studying tracheal epithelial dynamics will provide a good indication of tissue as a regulator of physiological and pathological events in the respiratory system. As the airways of animals are exposed to different environments, their airway dynamics can potentially be altered. Therefore, the hypothesis is that the airways of pigs reared indoors have morphological differences compared to those reared outdoors. This study is aimed to determine differences within the airways of pigs reared indoors compared to those reared outdoors.

\section{MATERIALS AND METHODS}

\subsection{Animals and Housing}

The North Carolina Agriculture and Technical State University (NCAT) Institutional Animal Care and Use Committee (IACUC) approved all experimental procedures involving animals in this study and studies were performed in accordance with the Guide for the Care and Use of Agricultural animals in Agricultural Research and Teaching (FAO, 2010). All animals used in Trials 1, 3 and 3 were raised and housed at the Swine Research Unit located at NCAT. The Swine Research Unit includes a 250 -sow indoor commercial hog-rearing environment, as well as two hoop barns and a pastured hog operation for small-scale or limited-resource farmers making the transition to hog farming from tobacco or other crops. Animals were allowed to reach finishing stage (body weight of 19.1 kilograms) before airway tissues were harvested to allow adequate time for acclimation to housing type and stage consistency. At the end of each trial, animals were transported to a USDAapproval abattoir for processing. For comparisons, airways from market weight hogs were also collected from a USDA-approved abattoir in accordance with a NCAT IACUC-approved protocol.

\subsection{Experimental Design}

\section{Trial 1}

The objective of trial 1 was to evaluate the impact of indoor rearing on airway morphology and airway epithelial proteomes using three different breed types. Fifteen crossbred pigs (Tamworth X Berkshire (TB), Berkshire X Berkshire (BB) and Hereford X Berkshire (HB)) were selected from the NCAT Farm-Swine Research Unit. Trial 1 was conducted from September 2009 through March 2010. Pigs were maintained in environmentally controlled confinement housing pens with ad libitum feed and access to nipple drinkers. At approximately five to six months of age, pigs were transported to a USDA approved abattoir for harvest. Only fourteen tracheas were collected because one was obliterated during the dissection process and could not be used in study. The crossbred tracheal samples collected were grouped as follows: TB $(\mathrm{n}=5), \mathrm{BB}(\mathrm{n}=5)$ and $\mathrm{HB}(\mathrm{n}=4)$.

\section{Trial 2}

The objective of trial 2 was to evaluate the impact of confinement versus pasture-based rearing on the airways. Twenty-eight pigs, born and weaned from a confinement unit, were reared in a pasture-based setting during the months of June through November 2010. At approximately five to six months of age, pigs were transported to a USDA approved abattoir for harvest. All tracheal samples collected were evaluated as described below and stored for further processing. However, for trial 2, only fourteen of the outdoor tracheal samples were randomly selected and used. The fourteen tracheas that were collected from trial 1 were used within trial 2 to compare the differences between the two management styles and their impact on airway morphology.

\section{Trial 3}

The objective of trial 3 was to investigate the impact of management style on porcine airway dynamics while controlling variables such as body weight, breed and sex. Forty-eight crossbred piglets were selected (primarily Yorkshire and Landrace crossbred) from the 
NCAT Farm-Swine Research Unit. The trial was conducted from April through May 2011. After the weaning period (three weeks), twenty-four piglets remained within an indoor production setting and twenty-four piglets were placed within an outdoor production setting. All animals in the study were given numbered ear tags for identification. Body weights were determined and recorded weekly over a seven week period as previously described (Whitley et al., 2012). Final body weights were recorded on the day of tissue harvest; at approximately seven weeks of age.

Within the indoor setting, the twenty-four pigs were assigned to one of three nursery rooms which were environmentally controlled and maintained at 22.2 to $25.6^{\circ} \mathrm{C}$. Each nursery room contained a total of eight pigs. Within each room, the pigs were kept in $1.2 \times 3.0$ meter pens with four pigs per pen. Metal slats within the pens allowed for manure disposal. Pigs were fed 8.2 kilograms of a normal NRCS-based growing pig diet daily and had access to nipple drinkers.

Within the outdoor setting, pigs were divided into three pasture areas $(15.2 \times 29.0$ meters $)$ with eight pigs each. Pigs were fed 10.9 kilograms of a normal growing feeding diet daily and had access to nipple drinkers. These animals tended to eat more during the study; therefore, animals from this treatment group were provided more feed. The trial lasted seven weeks. Figure 1 shows the experimental design of the two management environments used in trial 3. Animals were kept in their designated areas for seven weeks. The outdoor animals (Fig. 1A) were provided with feed (F), English Hut for shelter (S) and Water (W). Indoor pigs (Fig. 1B) were separated further into two groups of four in adjacent pens with their own feeder and water (F).

\subsection{Airway Measurements}

At harvest, the tracheal portion of the respiratory tract was collected by severing the connective tissue beneath the larynx (anterior to the first cartilaginous ring of the trachea) and directly above the point of bronchial branching into the lungs. Excess connective tissue, lymph nodes, heart and lung tissue were removed from specimens using a scalpel. Following dissection, each trachea was placed, separately, into a zip-top plastic bag and kept on wet ice during transportation. In the laboratory airway measurements were taken on the airways.

On the day of harvest, several parameters were measured including tracheal length, total tracheal diameter, tracheal lumen (inside) diameter and tracheal weight. Figure 2 displays a model of the measurements recorded during this data collection. Tracheal length was measured from the top of the arrow right below the larynx to the beginning of the principal bronchus (Fig. 2A). Tracheal diameter was measured in two directions on a longitudinal and latitudinal plane (Fig. 2B). Tracheal lumen was measured in two directions, similar to the tracheal diameter. However, this pertains to the airspace within the tracheal (Fig. 2C). Whole tracheal weight was recorded before the one to two inch segment was removed and weighed (Fig. 2D). A 1-2-inch section was removed from the sub laryngeal region of the windpipe and stored in- $80^{\circ} \mathrm{C}$ for further processing (Fig. 2E).

\subsection{Histological Preparation and Evaluations}

For histological analysis, tracheas were collected from market weight adult pigs (at least $125 \mathrm{~kg}$ ) from a local abattoir. At harvest, tracheas were kept on ice during transport. At the laboratory, $2.5 \mathrm{~cm}$ sub laryngeal section was dissected from the entire trachea. These sections were transferred from ice to $4 \%$ par formaldehyde and were allowed to fix for approximately $4 \mathrm{~h}$ at $4^{\circ} \mathrm{C}$. Afterwards, the tracheas were transferred to a $30 \%$ sucrose solution and kept at $4^{\circ} \mathrm{C}$ for at least $1-2$ days. Tissue was considered post fixed once the trachea sank to the bottom of the tube and was stored at $-80^{\circ} \mathrm{C}$.

Tracheas were thawed and dissected into four even cross sections. One of these quarters was sectioned on a Leica CM3050S cryostat (Nussloch, Germany). Briefly, the section was mounted onto a stage with either 22 Blue (FSC) compound (Leica, Nussloch Germany) or O.C.T. Compound (Sakura Torrance, CA). Six micron sections were cut with the object temperature of $-20^{\circ} \mathrm{C}$ and a chamber temperature of $-25^{\circ} \mathrm{C}$. Sections were mounted onto positively charged glass microscope slides, Snowcoat X-tra (Leica, Nussloch Germany). Slides not immediately stained with Periodic Acid Schiff's Stain (PAS) (Polysciences, Inc. Warrington, PA) were stored at $-80^{\circ} \mathrm{C}$ until staining could be performed.

To visualize the goblet cells, PAS was used to stain the sections of trachea. Staining was done at room temperature. Briefly, the tissue was incubated in $0.5 \%$ Periodic Acid for $5 \mathrm{~min}$, followed by three washes with distilled water. Next, the tissue was immersed in Schiff's reagent for $15 \mathrm{~min}$, washed with two $1 \mathrm{~min}$ incubations of $0.55 \%$ Potassium Metabisulfite, followed by a $10 \mathrm{~min}$ wash in running water. The sections were counter stained for $30 \mathrm{sec}$ with Acidified Harris Hematoxyline. Excess stain was removed by rinsing in running water. After staining, the tissue was dehydrated through 70, 95 and $100 \%$ ethanol, with final incubations through two sets of xylene. The stained tissue was then mounted under coverslips with Permount (Fisher Scientific, Fair Lawn, NJ). Goblet Cells were visualized and counted at $1000 \times$ objective on a Leica DME light microscope (Nussloch, Germany). 


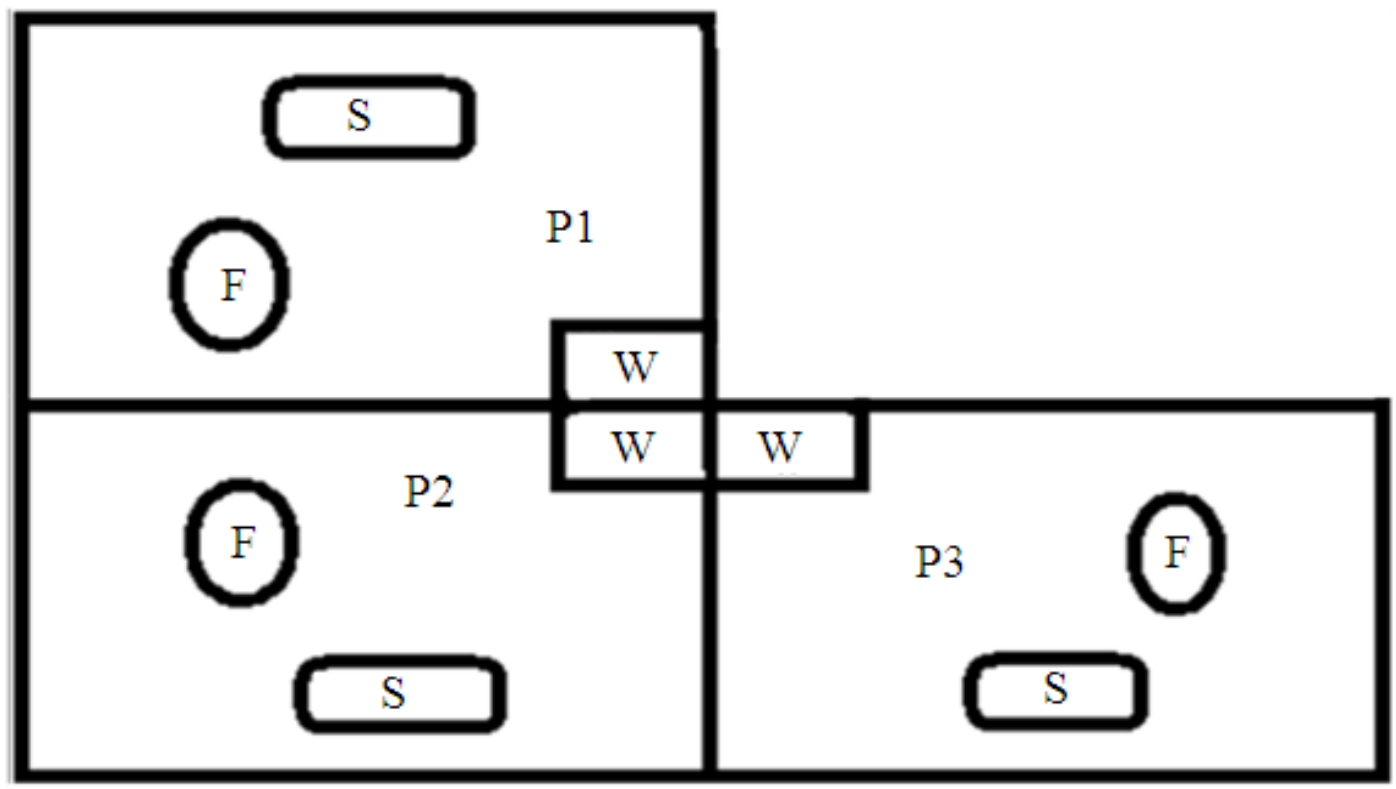

\section{Outdoor}

(A)

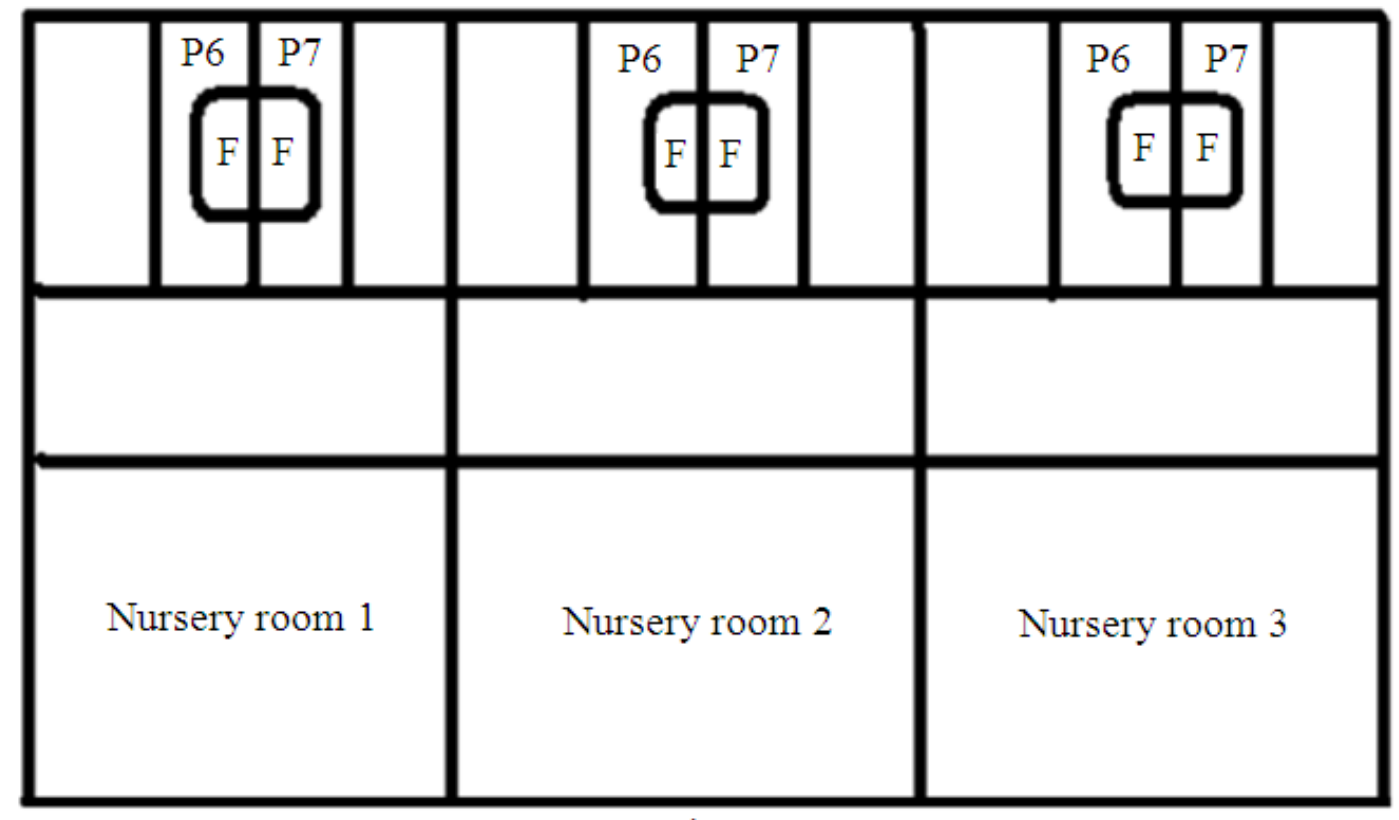

Indoor

(B)

Fig. 1. Outdoor and indoor experimental design for Trial III.Within the outdoor setting, pigs were divided into three pasture areas $(15.2 \times 29$ meters) with eight pigs each. Pigs were fed a normal growing feeding diet daily and had access to nipple drinkers. The outdoor animals (left) were provided feed (F), English Hut for shelter (S) and water (W). Indoor pigs (right) were separated further into two groups of four in adjacent pens with their own feeder and water $(\mathrm{F})$. The trial lasted seven weeks 

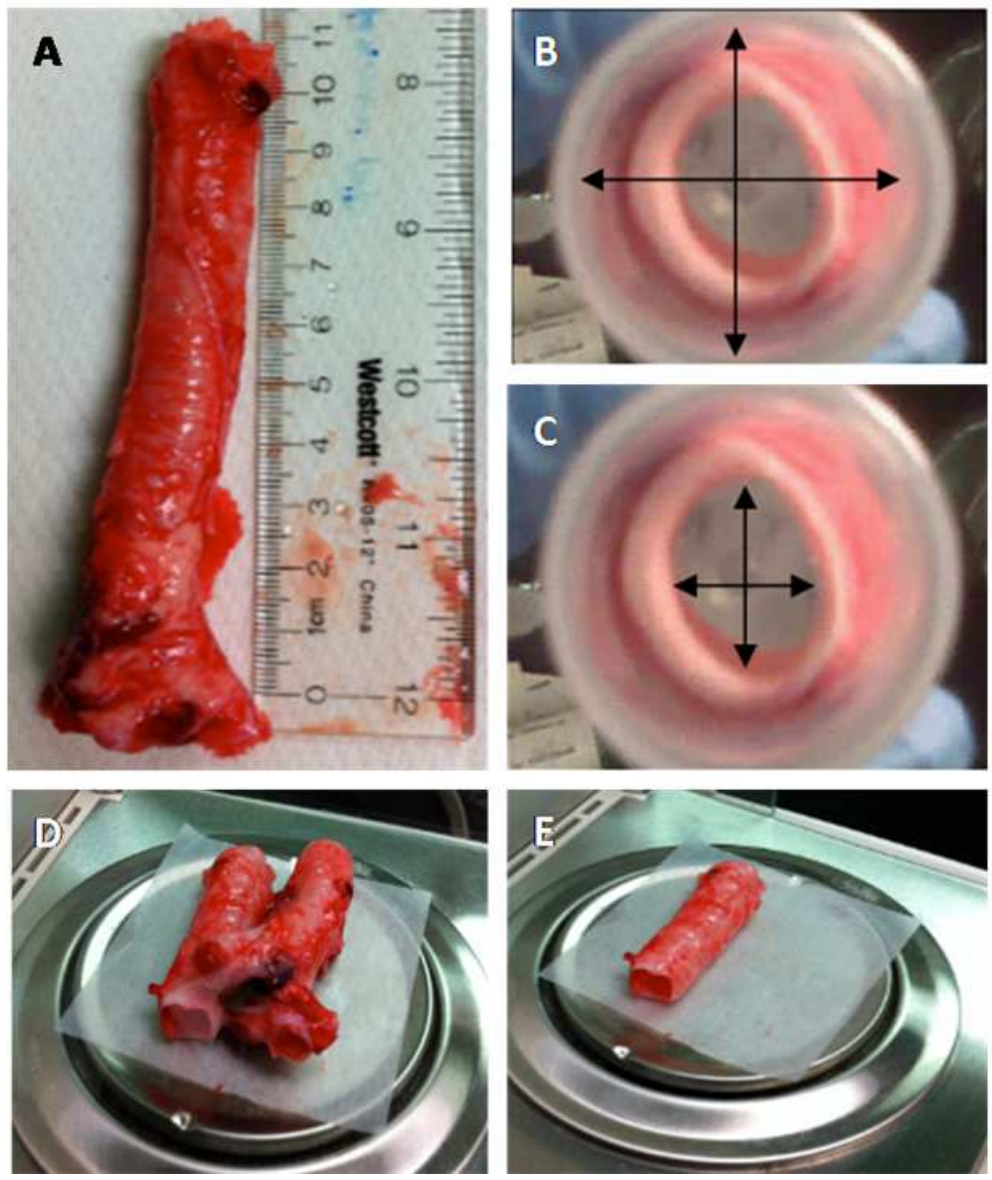

Fig. 2. Tracheal segments for airway measurements. A, Tracheal length was measured from the top of the arrow right below the larynx to the beginning of the primary bronchus. B, Total tracheal diameter was measured in two directions on a longitudinal and latitudinal plane. C, Tracheal lumen was measured in two directions, similar to the tracheal diameter. D, Whole tracheal weight was recorded before a one to two-inch segment was removed and weighed. E, Sublaryngeal tracheal sections (1-2 inch) utilized for histological analysis or stored at $-80^{\circ} \mathrm{C}$ for further processing

\subsection{Statistical Analyses}

Three distinct experimental trials were conducted to evaluate the effect of swine management programs on the large airways of the pig. Statistical models employed for each trial are described below. The analysis employed to evaluate goblet cell densities is described here as well.

\section{Trial 1}

All data were analyzed utilizing the GLM procedure (SAS Institute Inc., Cary, NC). The breed of pig served as the main effect and the statistical model included breed only. Recorded airway measurements were analyzed by a one-way Analysis of Variance (ANOVA) at a p-value of 0.05 to detect significant difference between means followed by lsmeans PDIFF option as applicable to compare breed type.

\section{Trial 2}

All data were analyzed utilizing the GLM procedure (SAS Institute Inc., Cary, NC). The pigs served as the experiment unit. The statistical model included housing only. Airway metrics were analyzed by a one-way ANOVA at a p-value of 0.05 to detect significant difference between means followed by lsmeans PDIFF option as applicable to compare all parameters measured within the indoor and outdoor environments. 


\section{Trial 3}

All data were analyzed utilizing the GLM procedure (SAS Institute Inc., Cary, NC). Due to the mixing of pigs in pens after weaning, the pens served as the experimental units. The statistical model included housing, pen and pen by housing interaction. Airway metrics were analyzed by a two-way ANOVA at a p-value of 0.05 to detect significant difference between means followed by lsmeans PDIFF option as applicable to compare all groups. Repeated body weight measurement overtime was performed to evaluate body weight variation within each management style. A correlation analysis was used to determine a possible association between body weight and airway size.

\subsection{Histological Analysis}

Datawas analyzed utilizing GraphPad Prism 5.0 (GraphPad Software, Inc., La Jolla, CA) where appropriate. Trachea goblet cell populations of indoor versus outdoor pigs were compared using a one way ANOVA, with a Bonferroni multiple comparison posttest. A p-value of 0.05 was employed to determine significance.

\section{RESULTS}

\subsection{Analysis of Airway Metrics Reveal Distinct Airway Features of Animals Reared Indoors}

It is well established that exposure to CAFO-style production facilities leads to increased respiratory complications in individuals who work within CAFOs (Heederik et al., 2007; May et al., 2012). Two respiratory complications that are commonly observed and are associated with morphological changes within the airways include asthma and chronic bronchitis. Therefore, to better understand these potential health risks, it is essential to determine whether differences are present when animals are raised indoors versus outdoors along with the possible impact each environment has on the airway. To meet this aim, the impact of indoor and outdoor swine production styles on airway morphology was evaluated.

In trial 1, tracheal diameters and lumens of indoorraised animals with different genetic backgrounds were evaluated for possible differences. Results indicated no differences exist between the different breed types (Fig. 3A, B). Therefore, to compare animals raised indoors versus those raised outdoors, a second experimental trial was performed using animals reared outdoors. The data collected from this trial was then compared to the data collected from trial 1 on indoor raised animals. To compare the data from these two trials, a one-way ANOVA was performed with a post-test of lsmeanPDIFF option as applicable. When comparing the two, it was found that tracheal diameters were greater for indoor samples compared to the outdoor, $\mathrm{p}=0.0014$. A difference was also present when comparing the lumen diameter of animals reared indoors and outdoors ( $\mathrm{p}$-value $=$ 0.0001). Figure 4 displays the tracheal diameter (Fig. 4A) and tracheal lumen (Fig. 4B) data collected for the two swine production operations.

Since data comparisons were made using tracheas harvested from animals in the first two trials, trial 3 was performed to compare indoor and outdoor housing types using animals that were reared at the same time. During this trial, association between airway size and body weight was also evaluated. Animals were either housed in 1). A pen located within one of three rooms (indoor) or 2.) One of three pastures (outdoor).

As in trials 1 and 2, airway metrics were collected in trial 3. When comparing the indoor and outdoor swine in trial 3, no differences were observed regarding tracheal diameter and lumen $(p>0.05)$ as listed in Table 1. Figure 5 displays the results from the two-way ANOVA that was completed on the tracheal diameters and lumens of the animals used in trial 3. The mean of each treatment group was used to plot the data. As noted in Table 1, a significant pen by housing interaction for the average tracheal lumen was present. This interaction suggests the possibility of a confounding factor that is contributing to the size of the airway and causing the differences. Bodyweight was also included into the statistical model as a covariate, but no significant differences were present (Table 1). Therefore, for this study, the results illustrated no correlation between the size of the animal and the size of the airway.

\subsection{Body Weight Variation in Pigs Reared Outdoors}

Trial 3 was conducted in April 2011 to May 2011. Within this two month period, bodyweights of all pigs were recorded. The average bodyweight within each pen/pasture are represented in Fig. 6. Results display a higher variation of bodyweights among the outdoor animals when compared to the indoor animals. 
Table 1. Airway measurements, tracheal diameter and lumen trial 3

\begin{tabular}{llllll}
\hline & Tracheal diameter & & & \\
Source & DF & Type I SS & Mean square & F Value & Pr $>$ F \\
\hline Pen & 2 & 0.07875000 & 0.03937500 & 0.99 & 0.3795 \\
Housing & 1 & 0.05333333 & 0.05333333 & 1.34 & 0.2531 \\
Pen*housing & 2 & 0.20041667 & 0.10020833 & 2.53 & 0.0924 \\
Bodyweight & 1 & 0.00039017 & 0.00039017 & 0.01 & 0.9215 \\
Tracheal lumen & & & & & \\
Pen & 2 & 0.08791667 & 0.04395833 & 1.60 & 0.2149 \\
Housing & 1 & 0.03000000 & 0.03000000 & 1.09 & 0.3027 \\
Pen*housing & 2 & 0.19625000 & 0.09812500 & 3.56 & 0.0374 \\
Bodyweight & 1 & 0.03357637 & & 1.22 & 0.2759 \\
\hline
\end{tabular}
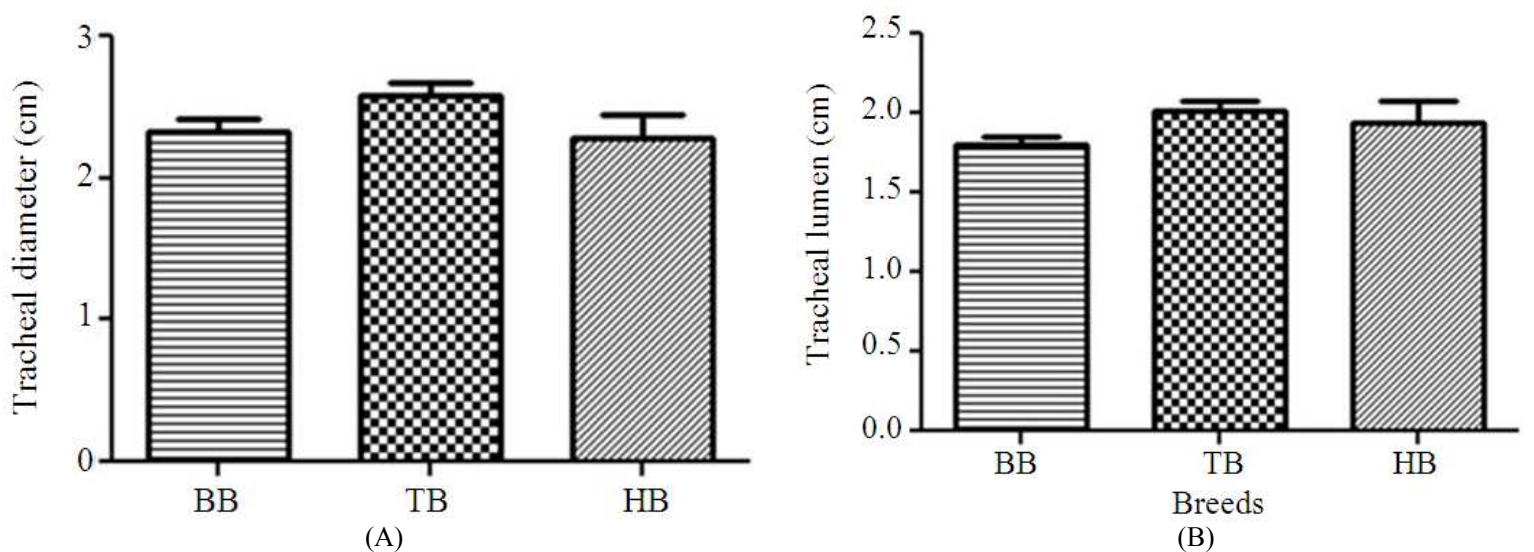

(B)

Fig. 3. Trial 1 tracheal diameter and lumen measurements amongst genetically distinct pigs reared indoors. Airway measurements among breed type were analyzed. Tamworth X Berkshire $(T B, n=5)$, Berkshire X Berkshire $(B B, n=5)$ and Hereford $X$ Berkshire $(\mathrm{HB}, \mathrm{n}=4)$ pigs were used. Tracheal diameter $(\mathrm{p}=0.1)$ and tracheal lumen $(\mathrm{p}=0.2)$ were not influenced by breed type. Data are shown as means \pm SEM

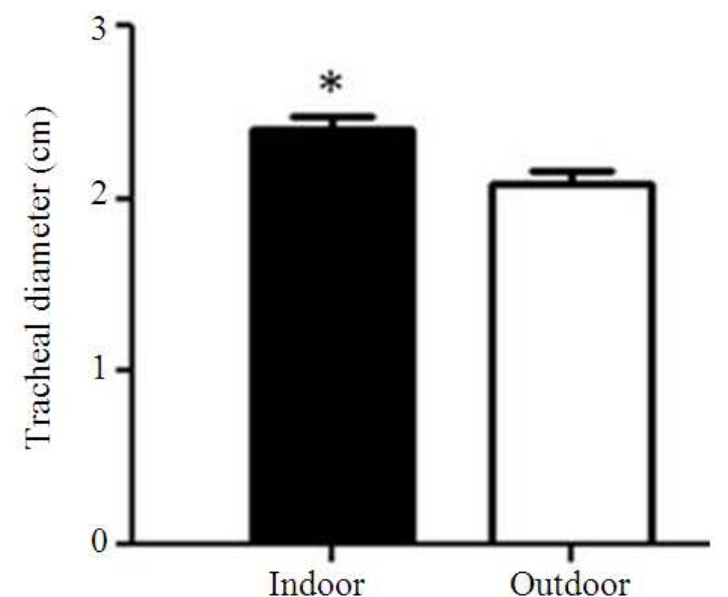

(A)

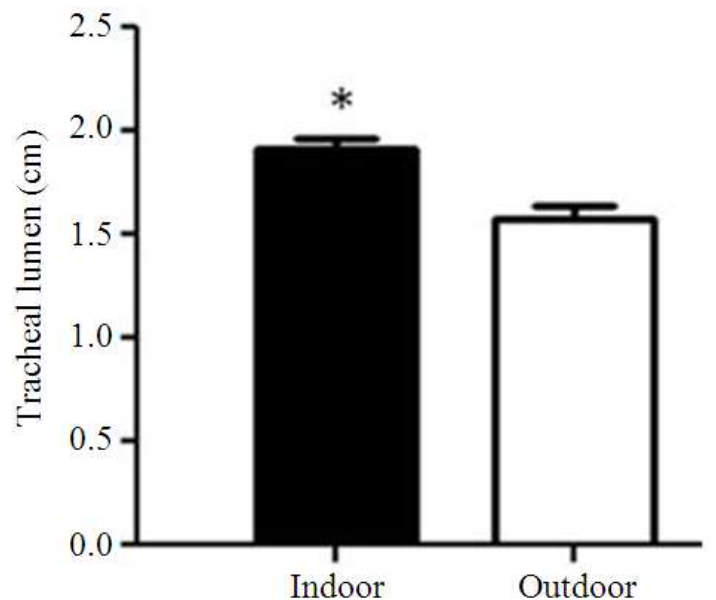

(B)

Fig. 4. Trial 2, Tracheal diameter and tracheal lumen measurements among pigs reared indoors (trial 1) and outdoors (trial 2). Indoor reared pigs had larger tracheal diameters and lumens than outdoor reared animals $\mathrm{P}=0.0001$ and 0.0014 , respectively. Data are shown as means \pm SEM. There were 14 indoor and 14 outdoor reared animals 

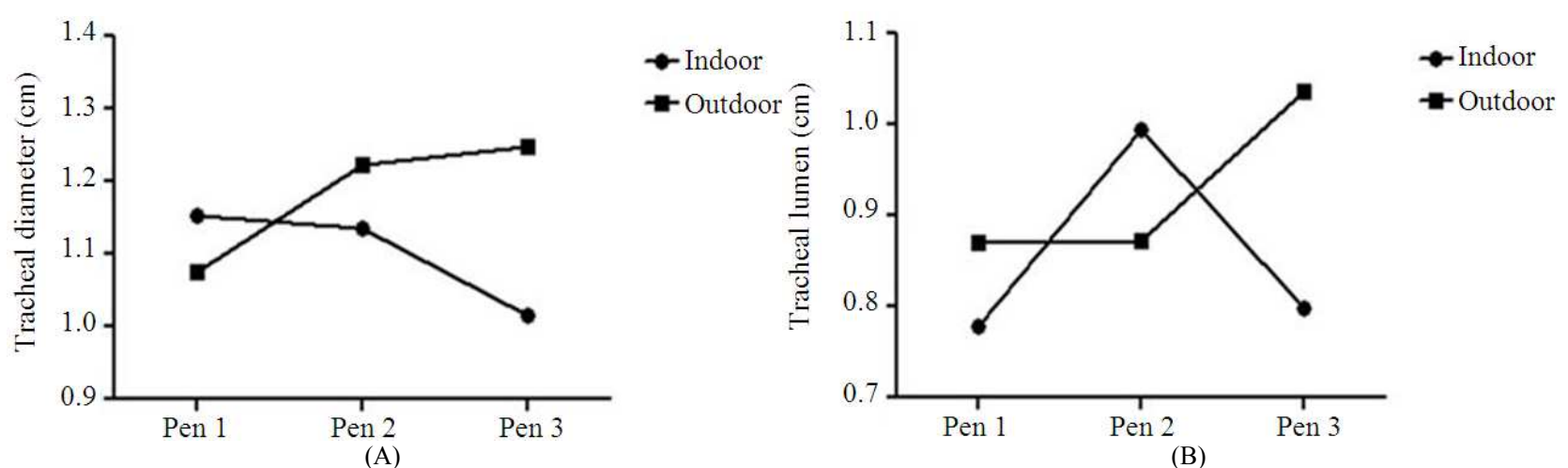

Fig. 5. Trial 3 average tracheal diameter and lumen measurements of indoor and outdoor reared animals, A average tracheal diameter, B average tracheal lumen. There is a pen by housing interaction for lumen diameter $(\mathrm{p}=0.03) . \mathrm{n}=24$ each

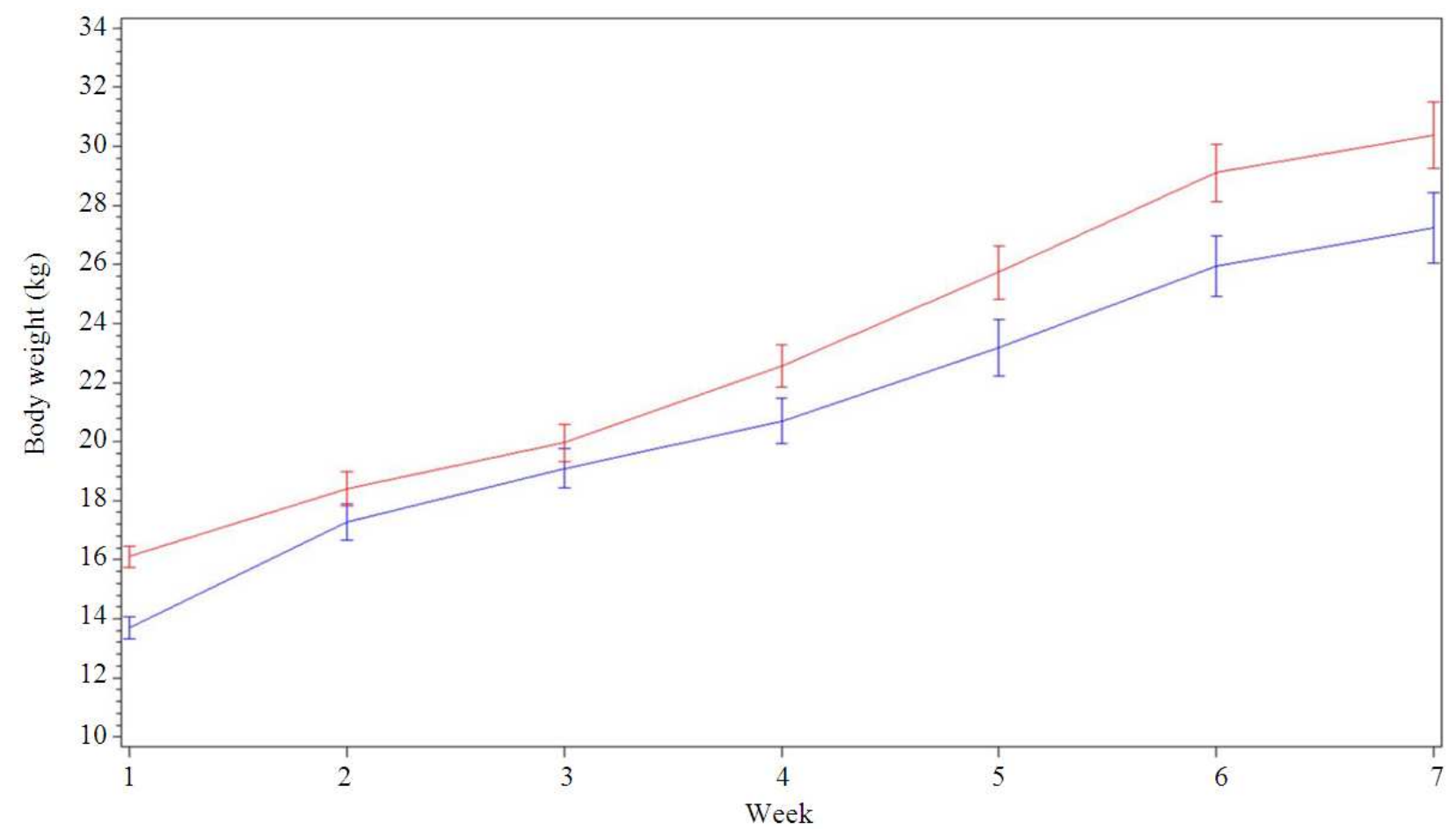

Fig. 6. Trial 3 indoor and outdoor animal body weight repeated measurement overtime. Weekly bodyweights were recorded in kilograms and the average bodyweight within each housing type was plotted. Confinement animals are represented in blue and the pasture-raised pigs are represented as red. Data are plotted as means \pm SEM. There were 24 animals in each group

\subsection{Goblet Cell Density of Porcine Tracheal Epithelia}

Changes in the presence and distribution of goblet cells within the airway epithelium provide insight into respiratory health and disease (Chen et al., 1991). In the present study, analysis of airway epithelia from adultindoor rearedpigs had a goblet cell population of 960.2 cells per quarter cross section slice [Indoor $=$ 102.7 cells $/ \mathrm{mm}$, Outdoor $=38.7$ cells $/ \mathrm{mm}]$. Outdoor reared pigs had an average of 667.4 per section. Figure 7 depicts a representative PAS stained cross section of indoor (A) and outdoor (B) porcine tracheas. 

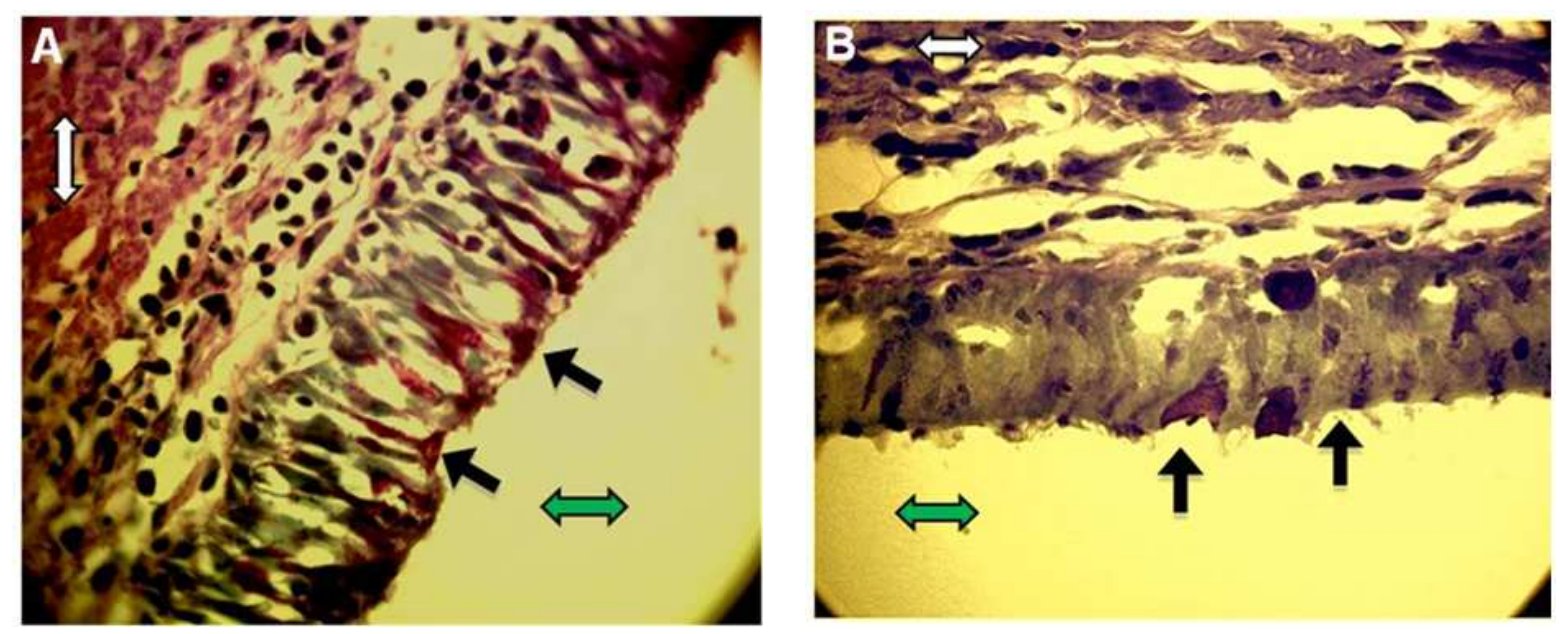

Fig. 7. Periodic Acid Schiff stained sections of porcine tracheas. Images of histological sections from airways of A, indoor and B, outdoor reared animals. Goblet cells are present in the epithelial layer of the trachea, bordering the lumen. Black arrows point to goblet cells; white arrows denote connective tissue and the green arrows show the lumen of the trachea. Images are representative of three for each group. Magnification $=1000 \mathrm{X}$

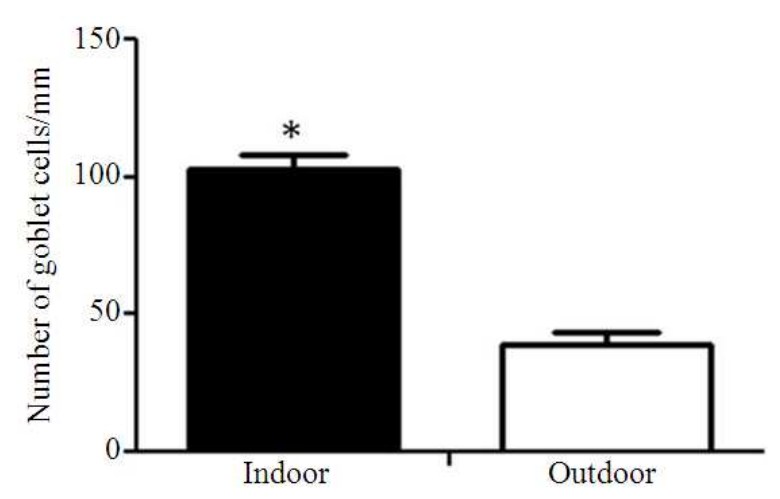

Fig. 8. Tracheal epithelia of pigs reared indoors are densely packed with goblet cells. *, p-value $<0.0001$. Data presented as mean \pm SEM. $\mathrm{n}=3$

Results were subjected to a one way ANOVA, with Bonferroni's multiple comparison test. The tracheas of indoor reared pigs had a significantly higher population of goblet cells ( $\mathrm{p}$-value $<0.0001$ ), when compared to outdoor reared pigs (Fig. 8).

\section{DISCUSSION}

Over the years, swine producers have transitioned their outdoor operations indoors to produce greater amounts of pork. Of the two primary types of porcine operations, confinement housing is typically implicated for its impact on human respiratory health. Many studies report the deleterious effects of reduced air quality in SCF on the respiratory health of humans (Alterman et al., 2008; Letourneau et al., 2010). Poor air quality in swine confinement housing can be caused by an array of contributing factors such as bacterial agents, atmospheric emissions such as ammonia-nitrogen and hydrogen sulfide (Blunden et al., 2008; Heber et al., 2006) and particulate matter (Cai et al., 2006). Few studies have focused on evaluating the impact of indoor versus outdoor production on the airway morphology and function of pigs. Therefore, in the present study, three experimental trials were conducted and histological samples were prepared to evaluate the morphological, physiological and proteomic differences in the airways of pigs raised in either an indoor or outdoor production setting. From the data collected, it was determined that the production environment may cause subtle, but distinct differences in the airways of the animals reared within them.

In trial 1, airway morphologies of tracheal regions were determined by airway measurements. No real difference was found when using genetic background as the main effect. In trial 2, only outdoor pigs were used, but comparisons were made with indoor pigs from trial 1. Significant differences were observed in the tracheal diameters found between trial 1 (indoor) and trial 2 (outdoor) pigs. Indoor animals were shown to have larger tracheal diameters and lumens than the outdoor animals. These distinctions may be due to differences in 
airway adaptation responses among the two groups. It has been shown that repeat expsoure to CAFO dust alters airway reactivity, resulting an adaptive type of response in farmers (Sundblad et al., 2009) and mice (Poole et al., 2009). However, for this study, the indoor animals were five to six months old at the time of harvest and were kept exclusively indoors which could have resulted in them generating a level of tolerance to the confined environment. However, the significance of these differnences are unclear.

Trial 3 tested main effects of body weight, pen and housing environment and the interaction. No differences were present between the two environments for the tracheal and lumen diameter, but an interaction effect for pen by housing did exist. This interaction indicates a potential effect may be caused by pens within a production style. It was also initially suspected that bodyweight could influence trachea or airway size. However, according to this present study, trial 3 bodyweights of pigs at 7 weeks of age did not influence the size of the airway. Other factors, such as the age of the animals, may have caused the pen and housing interaction to be significant. In a recent report, airway reactivity had been observed in healthy young rats at 6 weeks old compared to 21 week old rats (Lee et al., 2007). Lee et al. (2007) suggested young rats are more responsive to cholinergic stimulation in vivo, which appeared to be partially due to greater cholinergic responsiveness of the airway smooth muscle. In accordance, human studies where infant, young and old subjects were exposed to methacholine have shown that infants and young subjects responded more intensely to the exposure (Kott et al., 2002). However, it is reasonable to accept that the anatomical airway features of young animals, whether they are pigs, rats or humans, are smaller and therefore potentially more reactive than adult airways. This data suggests that age can influence how animals respond to different environmental exposures even though the pigs in trial 1 and 2 were of similar age. Genetic variation may also explain differences observed in airway measurements. Although trial 1 tested breed type, the number of experimental units may need to be increased to illustrate appreciable difference. It is plausible that genetic background can mediate the anatomical size of the airway. The pigs used in trial 3 were still relatively young and may not have been influenced significantly by their environmental setting.

In trial 3 , body weight was measured repeatedly over seven weeks. Outdoor animals had a larger variation of body weights than the indoor animals. This finding can be due to many factors such as social behavior, climate, genetics, nutrition and housing as discussed by Honeyman (2005). The variation in body weights can be due to stress. Rutherford et al. (2006) conducted a study where juvenile male pigs were exposed to social and environmental stress routines. Over the stressor period, weight gain was significantly reduced in stress treatment pigs when compared to the control pigs (Rutherford et al., 2006). Trial 3 was conducted in April 2011 to May 2011 when the outdoor temperatures remained at 26.7 to $32.2^{\circ} \mathrm{C}$. During this time, some pigs may have been more susceptible to temperature stress which may have resulted in the variation observed for outdoor pigs. The litter size or the number of pigs within each pen/pasture can also make a difference in feed consumption. In the beginning of trial 3 , outdoor animals were only receiving $8.16 \mathrm{kgs}$ of feed per day. After the first week, feed was increased to $10.89 \mathrm{kgs}$ per day. The outdoor animals had eight pigs per pasture, tended to eat more and had additional outdoor feed possibilities. However, the indoor animals were given $8.16 \mathrm{kgs}$ of feed a day, but only had four animals per feeder and continued to have less variation in body weights. Feed intake can also be influenced by the season. According to Oliveira et al. (2009), during the months of September and December, finishing pig feed intake tends to increase more than when compared to January to April and May to August.

The histological findings show significantly more goblet cells in the tracheal epithelia of indoor reared pigs compared to outdoor reared pigs. This result indicates a clear difference in airway epithalial tissue organization of indoor reared pigs compared to outdoor reared pigs. The cytoplasm of porcine tracheal goblet cells contains several clusters of mucin granules (Adler et al., 1982); mucin is the protein component of mucus. The airway epithelium is a dynamic tissue capable of undergoing reversible cellular adaptations, including goblet cell hyperplasia, in response to a myriad of stimuli (Chen et al., 1991; Rock et al., 2010). An increase in the number of goblet cells essentially translates to a higher capacity for mucus production and secretion. The significance of differences in goblet cell density observed in this study is unclear. These differences may reflect distinct goblet cell and/or mucus production needs influenced by housing type; however, this point has not been clarified. Thus, studies are necessary to evaluate the confounding factors of age, breed and body weight over a period of time and with regard to airway dynamics. 


\section{CONCLUSION}

The airway is a very dynamic organ that is equipped with rapid response mechanisms because of its constant exposure to the external environment. However, respiratory chronic protective functions can lead to pathological effects, tissue damage and terminally COPD. Therefore, the changes of airway structure are an essential indicator to respiratory health. This study of swine airways provides insight into the airway dynamics of pigs reared indoors and outdoors, as well as a potential model for evaluating exposures experienced by swine producers, workers and farm visitors. In conclusion, animals reared indoors may have display distinct tracheal and airway epithelial dynamics compared to those reared outdoors, which may, in part, explain their ability to live within confinement houses without any apparent complications; however, further studies are needed to support this conclusion.

\section{ACKNOWLEDGEMENT}

This project was supported by USDA National Institute of Food and Agriculture GrantsNC.X-255-5-11120-1 (to J.T.W.). S.G.P. was a National Science Foundation (NSF) North Carolina Louis Stokes Alliance for Minority Participation (NC-LSAMP) Fellow. This study constitutes a portion of a thesis by the first author, C.J.M, completed in partial fulfillment of the requirements for the Master of Science degree (NCAT). The authors thank T. Barrios for technical assistance with animal husbandry and R. C. Minor for critical reading of the manuscript.

\section{REFERENCES}

Adler, K.B., D.H. Hardwick and J.E. Craighead, 1982. Porcine tracheal goblet cell ultrastructure: A threedimensional reconstruction. Exp. Lung Res., 3: 6980. PMID: 7060544

Alterman, T., A.L. Steege, J. Li, M.R. Petersen and C. Muntaner, 2008. Ethnic, racial and gender variations in health amongfarm operators in the United States. Annals Epidemiol., 18: 179-186. DOI: 10.1016/j.annepidem.2007.11.014

Blunden, J., V.P. Aneja and P.W. Westerman, 2008. Measurement and analysis of ammonia and hydrogen sulfide emissions from a mechanically ventilated swine confinement building in North Carolina. Atmospheric Environ., 42: 3315-3331. DOI: 10.1016/j.atmosenv.2007.06.040
Cai, L., J.A. Koziel, Y.C. Lo and S.J. Hoff, 2006. Characterization of volatile organic compounds and odorants associated with swine barn particulate matter using solid-phase microextraction and gas chromatography-mass spectrometry-olfactometry. J. Chromatography A, 1102: 60-72. DOI: 10.1016/j.chroma.2005.10.040

Chen, W., M.R. Alley and B.W. Manktelow, 1991. Morphological and morphometric studies of the airways of sheep with acute airway hypersensitivity to inhaled Ascaris suum. Int. J. Exp. Pathol., 72: 543-51. PMID: 1742207

Cole, D., L. Todd and S. Wing, 2000. Concentrated swine feeding operations and public health:A review of occupational and community health effects. Environ. Health Perspectives, 108: 685-699. PMCID: PMC1638284

FAO, 2010. Guide for the Care and Use of Agricultural Animals Used in Agricultural Research and Teaching. 3rd Edn., Food and Agriculture Organization of the United Nations (FOA).

Heber, A.J., J.Q. Ni, T.T. Lim, P.C. Tao and A.M. Schmidt et al., 2006. Quality assured measurements of animal building emissions: gas concentrations. J. Air Waste Manage. Assoc., 56: 1472-1483. PMID:17063869

Heederik, D., T. Sigsgaard, P.S. Thorne, J.N. Kline and R. Avery et al., 2007. Health effects of airborne exposures from concentrated animal feeding operations. Environ. Health Perspect., 115: 298-302. DOI: $10.1289 / \mathrm{ehp} .8835$

Honeyman, M.S., 2005. Extensive bedded indoor and outdoor pig production systems in USA: Current trends and effects on animal care and product quality. Livestock Prod. Sci., 94: 15-24. DOI: 10.1016/j.livprodsci.2004.11.029

Kott, K.S., K.E. Pinkerton, J.M. Bric, C.G. Plopper and K.P. Avadhanam et al., 2002. Methacholine responsiveness of proximal and distal airways of monkeys and rats using videomicrometry. J. Applied Physiol., 92: 989-96. DOI: 10.1152/japplphysiol. 00415.2001

Lee, H.K. M.Y. Lim, S.M. Bok, E.S. Cho and E.M. Lee et al., 2007. Age differences in cholinergic airway responsiveness in relation with muscarinic receptor subtypes. Life Sci., 81: 204-209. DOI: 10.1016/j.lfs.2007.05.002 
Letourneau, V., B. Nehme, A. Meriaux, D. Masse and Y. Cormier et al., 2010. Human pathogens and tetracycline-resistant bacteria inbioaerosols of swine confinement buildings and in nasal flora of hog producers. Int. J. Hygiene Environ. Health, 213: 444-449. DOI: 10.1016/j.ijheh.2010.09.008

May, S., D.J. Romberger and J.A. Poole, 2012. Respiratory health effects of large animal feeding environments. J. Toxicol. Environ. Health B Crit. Rev., 15: 524-41. doi: 10.1080/10937404.2012.744288

Oliveira, J., E. Yus and F.J. Guitian, 2009. Effects of management, environmental and temporal factors on mortality and feed consumption in integrated swine fattening farms. Livestock Sci., 123: 221-229. DOI: 10.1016/j.livsci.2008.11.016

Poole, J.A., T.A. Wyatt, P.J. Oldenburg, M.K. Elliott and W.W. West et al., 2009. Intranasal organic dust exposure-induced airway adaptation response marked by persistent lung inflammation and pathology in mice. Am. J. Physiol. Lung Cell Mol. Physiol., 296: L1085-L1095. DOI: 10.1152/ajplung.90622.200.2008

Poole, J.A., T.D. LeVan, R.E. Slager, F. Qiu and L. Severa et al., 2007. Bronchodilator responsiveness in swine veterinarians. J. Agromedicine, 12: 49-54. PMID: 18086654

Radon, K., A. Schulze, V. Ehrenstein, R.T.V. Strien and G. Praml et al., 2007. Environmental exposure to confined animal feeding operations and respiratory health of neighboring residents. Epidemiology, 18: 300-300. PMID: 17435437
Rock, J.R., S.H. Randell and B.L.M. Hogan, 2010. Airway basal stem cells: A perspective on their roles in epithelial homeostasis and remodeling. Dis. Mod. Mechanisms, 3: 545-556. DOI: 10.1242/dmm.006031

Rutherford, K.M.D. M.J. Haskell, C. Glasbey and A.B. Lawrence, 2006. The responses of growing pigs to a chronic-intermittent stress treatment. Physiol. Behav., 89: 670-680. DOI: 10.1016/j.physbeh.2006.08.006

Sundblad, B.M., I. Von Scheele, L. Palmberg, M. Olsson and K. Larsson, 2009. Repeated exposure to organic material alters inflammatory and physiological airway responses. Eur. Respir. J., 34: 80-88. DOI: $10.1183 / 09031936.00105308$

Von Essen, S.G. and B.W. Auvermann, 2005. Health effects from breathing air near CAFOs for feeder cattle or hogs. J. Agromed., 10: 55-64. DOI: 10.1300/J096v10n04_08

Whitley, N., W.E.M. Morrow, M.T. See and S.H Oh, 2012. Comparison of growth performance of antibiotic-free Yorkshire crossbreds sired by Berkshire, large black and tamworth breeds raised in hoop structures. Asian-Aus. J. Anim. Sci., 25: 13511356. DOI: 10.5713 /ajas.2012.12162 